EPJ Web of Conferences 58, 01007 (2013)

DOI: $10.1051 /$ epjconf/20135801007

(C) Owned by the authors, published by EDP Sciences, 2013

\title{
Self Sustained Traversable Wormholes Induced by Gravity's Rainbow and Noncommutative Geometry
}

\author{
Remo Garattini ${ }^{1,2, a}$ \\ ${ }^{1}$ Università degli Studi di Bergamo, Facoltà di Ingegneria, \\ Viale Marconi,5 24044 Dalmine (Bergamo) ITALY \\ ${ }^{2}$ I.N.F.N. - sezione di Milano, Milan, Italy
}

\begin{abstract}
We compare the effects of Noncommutative Geometry and Gravity's Rainbow on traversable wormholes which are sustained by their own gravitational quantum fluctuations. Fixing the geometry on a well tested model, we find that the final result shows that the wormhole is of the Planckian size. This means that the traversability of the wormhole is in principle, but not in practice.
\end{abstract}

\section{Introduction}

In 1988 on the American Journal of Physics, M. S. Morris and K. S. Thorne published a paper entitled "Wormholes in spacetimes and their use for interstellar travel: A tool for teaching general relativity"[1]. Although the subject of the paper could be regarded as an argument of Science Fiction more than of Science, its impact on the scientific community was so amazing to open the doors to new investigations in Astrophysics, General Relativity and Quantum Gravity. In practice a traversable wormhole is a solution of the Einstein's Field equations, represented by two asymptotically flat regions joined by a bridge: roughly speaking it looks like a short-cut in space and time. To exist, traversable wormholes must violate the null energy conditions, which means that the matter threading the wormhole's throat has to be "exotic". Classical matter satisfies the usual energy conditions. Therefore, it is likely that wormholes must belong to the realm of semiclassical or perhaps a possible quantum theory of the gravitational field. Since a complete theory of quantum gravity has yet to come, it is important to approach this problem semiclassically. On this ground, the Casimir energy on a fixed background. has the correct properties to substitute the exotic matter: indeed, it is known that, for different physical systems, Casimir energy is negative. However, instead of studying the Casimir energy contribution of some matter or gauge fields to the traversability of the wormholes, we propose to use the energy of the graviton on a background of a traversable wormhole. In this way, one can think that the quantum fluctuations of the traversable wormholes can be used as a fuel to sustain traversability. Different contexts can be invoked to study self sustained traversable wormholes. In this paper, we review some aspects of self sustained traversable wormholes fixing our attention on Noncommutative geometry and Gravity's Rainbow.

\footnotetext{
ae-mail: remo.garattini@unibg.it
} 


\section{Self-sustained Traversable Wormholes}

In this Section we shall consider the formalism outlined in detail in Refs. [3, 4], where the graviton one loop contribution to a classical energy in a wormhole background is used. The spacetime metric representing a spherically symmetric and static wormhole is given by

$$
d s^{2}=-e^{2 \Phi(r)} d t^{2}+\frac{d r^{2}}{1-b(r) / r}+r^{2}\left(d \theta^{2}+\sin ^{2} \theta d \phi^{2}\right),
$$

where $\Phi(r)$ and $b(r)$ are arbitrary functions of the radial coordinate, $r$, denoted as the redshift function, and the form function, respectively [1]. The radial coordinate has a range that increases from a minimum value at $r_{0}$, corresponding to the wormhole throat, to infinity. A fundamental property of a wormhole is that a flaring out condition of the throat, given by $\left(b-b^{\prime} r\right) / b^{2}>0$, is imposed [1,2], and at the throat $b\left(r_{0}\right)=r=r_{0}$, the condition $b^{\prime}\left(r_{0}\right)<1$ is imposed to have wormhole solutions. Another condition that needs to be satisfied is $1-b(r) / r>0$. For the wormhole to be traversable, one must demand that there are no horizons present, which are identified as the surfaces with $e^{2 \Phi} \rightarrow 0$, so that $\Phi(r)$ must be finite everywhere. The classical energy is given by

$$
H_{\Sigma}^{(0)}=\int_{\Sigma} d^{3} x \mathcal{H}^{(0)}=-\frac{1}{16 \pi G} \int_{\Sigma} d^{3} x \sqrt{g} R
$$

where the background field super-hamiltonian, $\mathcal{H}^{(0)}$, is integrated on a constant time hypersurface. $R$ is the curvature scalar, and using metric (1), is given by

$$
R=-2\left(1-\frac{b}{r}\right)\left[\Phi^{\prime \prime}+\left(\Phi^{\prime}\right)^{2}-\frac{b^{\prime}}{r(r-b)}-\frac{b^{\prime} r+3 b-4 r}{2 r(r-b)} \Phi^{\prime}\right]
$$

We shall henceforth consider a constant redshift function, $\Phi^{\prime}(r)=0$, which provides interestingly enough results, so that the curvature scalar reduces to $R=2 b^{\prime} / r^{2}$. Thus, the classical energy reduces to

$$
H_{\Sigma}^{(0)}=-\frac{1}{2 G} \int_{r_{0}}^{\infty} \frac{d r r^{2}}{\sqrt{1-b(r) / r}} \frac{b^{\prime}(r)}{r^{2}} .
$$

A traversable wormhole is said to be "self sustained" if

$$
H_{\Sigma}^{(0)}=-E^{T T}
$$

where $E^{T T}$ is the total regularized graviton one loop energy. Basically this is given by

$$
E^{T T}=-\frac{1}{2} \sum_{\tau}\left[\sqrt{E_{1}^{2}(\tau)}+\sqrt{E_{2}^{2}(\tau)}\right]
$$

where $\tau$ denotes a complete set of indices and $E_{i}^{2}(\tau)>0, i=1,2$ are the eigenvalues of the modified Lichnerowicz operator

$$
\left(\hat{\triangle}_{L}^{m} h^{\perp}\right)_{i j}=\left(\triangle_{L} h^{\perp}\right)_{i j}-4 R_{i}^{k} h_{k j}^{\perp}+{ }^{3} R h_{i j}^{\perp},
$$

acting on traceless-transverse tensors of the perturbation and where $\Delta_{L}$ is the Lichnerowicz operator defined by

$$
\left(\triangle_{L} h\right)_{i j}=\triangle h_{i j}-2 R_{i k j l} h^{k l}+R_{i k} h_{j}^{k}+R_{j k} h_{i}^{k},
$$

with $\triangle=-\nabla^{a} \nabla_{a}$. For the background (1), one can define two r-dependent radial wave numbers

$$
k_{i}^{2}\left(r, l, \omega_{i, n l}\right)=\omega_{i, n l}^{2}-\frac{l(l+1)}{r^{2}}-m_{i}^{2}(r) \quad i=1,2,
$$


where

$$
\left\{\begin{array}{l}
m_{1}^{2}(r)=\frac{6}{r^{2}}\left(1-\frac{b(r)}{r}\right)+\frac{3}{2 r^{2}} b^{\prime}(r)-\frac{3}{2 r^{3}} b(r) \\
m_{2}^{2}(r)=\frac{6}{r^{2}}\left(1-\frac{b(r)}{r}\right)+\frac{1}{2 r^{2}} b^{\prime}(r)+\frac{3}{2 r^{3}} b(r)
\end{array}\right.
$$

are two r-dependent effective masses $m_{1}^{2}(r)$ and $m_{2}^{2}(r)$. When we perform the sum over all modes, $E^{T T}$ is usually divergent. In Refs. [3, 4] a zeta regularization and a renormalization have been adopted to handle the divergences. In this paper, we will consider the effect of the Noncommutative geometry and Gravity's Rainbow on the graviton to one loop.

\section{Noncommutative Geometry and Gravity's Rainbow at work on a Traversable Wormhole Background}

One of the purposes of Eq.(3) is the possible discovery of a traversable wormhole with the determination of the shape function. Nevertheless, another strategy can be considered if we fix the wormhole shape to be traversable, at least in principle. One good candidate is

$$
b(r)=r_{0}^{2} / r,
$$

which is the prototype of the traversable wormholes[1]. Plugging the shape function (9a) into Eq.(3), we find that the left hand side becomes

$$
H_{\Sigma}^{(0)}=\frac{1}{2 G} \int_{r_{0}}^{\infty} \frac{d r r^{2}}{\sqrt{1-r_{0}^{2} / r^{2}}} \frac{r_{0}^{2}}{r^{4}}
$$

while the right hand side is divergent. To handle with divergences, we have several possibilities. In this paper we adopt and compare two schemes: the Noncommutative scheme and the Gravity's Rainbow procedure. Beginning with the Noncommutative scheme, we recall that in Ref.[6], we used the distorted number of states

$$
d n_{i}=\frac{d^{3} \vec{x} d^{3} \vec{k}}{(2 \pi)^{3}} \exp \left(-\frac{\theta}{4}\left(\omega_{i, n l}^{2}-m_{i}^{2}(r)\right)\right), \quad i=1,2
$$

to compute the graviton one loop contribution to a cosmological constant. The distortion induced by the Noncommutative space time allows the right hand side of Eq.(3) to be finite. Indeed, plugging $d n_{i}$ into Eq.(3), one finds that the self sustained equation for the energy density becomes

$$
\frac{3 \pi^{2}}{G r_{0}^{2}}=\int_{0}^{+\infty} \sqrt{\left(\omega^{2}+\frac{3}{r_{0}^{2}}\right)^{3}} e^{-\frac{\theta}{4}\left(\omega^{2}+\frac{3}{r_{0}^{2}}\right)} d \omega+\int_{1 / r_{0}}^{+\infty} \sqrt{\left(\omega^{2}-\frac{1}{r_{0}^{2}}\right)^{3}} e^{-\frac{\theta}{4}\left(\omega^{2}-\frac{1}{r_{0}^{2}}\right)} d \omega,
$$

where we have used the shape function $(9 a)$ to evaluate the effective masses (8). If we define the dimensionless variable

$$
x=\frac{\theta}{4 r_{0}^{2}}
$$

Eq.(12) leads to $\left(G=l_{P}^{2}\right)$

$$
\frac{3 \pi^{2} \theta}{l_{P}^{2}}=F(x)
$$


where

$$
F(x)=\left((1-x) K_{1}\left(\frac{x}{2}\right)+x K_{0}\left(\frac{x}{2}\right)\right) \exp \left(\frac{x}{2}\right)+3\left((1+3 x) K_{1}\left(\frac{3 x}{2}\right)+3 x K_{0}\left(\frac{3 x}{2}\right)\right) \exp \left(-\frac{3 x}{2}\right) .
$$

$F(x)$ has a maximum for $\bar{x}=0.24$, where

$$
\frac{3 \pi^{2} \theta}{l_{P}^{2}}=F(\bar{x})=2.20
$$

This fixes $\theta$ to be

$$
\theta=\frac{2.20 l_{P}^{2}}{3 \pi^{2}}=7.43 \times 10^{-2} l_{P}^{2}
$$

and

$$
r_{0}=0.28 l_{P}
$$

As regards Gravity's Rainbow, as shown in Ref.[8], the self sustained equation (3) becomes

$$
\frac{b^{\prime}(r)}{2 G g_{2}\left(E / E_{P}\right) r^{2}}=\frac{2}{3 \pi^{2}}\left(I_{1}+I_{2}\right)
$$

where

$$
I_{1}=\int_{E^{*}}^{\infty} E \frac{g_{1}\left(E / E_{P}\right)}{g_{2}^{2}\left(E / E_{P}\right)} \frac{d}{d E}\left(\frac{E^{2}}{g_{2}^{2}\left(E / E_{P}\right)}-m_{1}^{2}(r)\right)^{\frac{3}{2}} d E
$$

and

$$
I_{2}=\int_{E^{*}}^{\infty} E \frac{g_{1}\left(E / E_{P}\right)}{g_{2}^{2}\left(E / E_{P}\right)} \frac{d}{d E}\left(\frac{E^{2}}{g_{2}^{2}\left(E / E_{P}\right)}-m_{2}^{2}(r)\right)^{\frac{3}{2}} d E .
$$

Eq.(18) is finite for appropriate choices of the Rainbow's functions $g_{1}\left(E / E_{P}\right)$ and $g_{2}\left(E / E_{P}\right)$. Fixing the shape function as in Eq. $(9 a)$ and assuming $g_{2}\left(E / E_{P}\right)=1$, to avoid Planckian distortions in the classical term, we find

$$
I_{1}=3 \int_{\sqrt{3 / r_{t}^{2}}}^{\infty} \exp \left(-\alpha E^{2} / E_{P}^{2}\right) E^{2} \sqrt{E^{2}-\frac{3}{r_{t}^{2}}} d E
$$

and

$$
I_{2}=3 \int_{0}^{\infty} \exp \left(-\alpha E^{2} / E_{P}^{2}\right) E^{2} \sqrt{E^{2}+\frac{1}{r_{t}^{2}}} d E,
$$

where we have also fixed $g_{1}\left(E / E_{P}\right)=\exp \left(-\alpha E^{2} / E_{P}^{2}\right)$ with $\alpha$ variable.

Now, in order to have only one solution with variables $\alpha$ and $r_{t}$, we demand that

$$
\frac{d}{d r_{t}}\left[-\frac{1}{2 G} \frac{1}{r_{t}^{2}}\right]=\frac{d}{d r_{t}}\left[\frac{2}{3 \pi^{2}}\left(I_{1}+I_{2}\right)\right],
$$

which takes the following form after the integration $\left(G^{-1}=E_{P}^{2}\right)$

$$
1=\frac{1}{2 \pi^{2} x^{2}} f(\alpha, x)
$$


where $x=r_{0} E_{P}$ and where

$f(\alpha, x)=\exp \left(\frac{\alpha}{2 x^{2}}\right) K_{0}\left(\frac{\alpha}{2 x^{2}}\right)-\exp \left(\frac{\alpha}{2 x^{2}}\right) K_{1}\left(\frac{\alpha}{2 x^{2}}\right)+9 \exp \left(-\frac{3 \alpha}{2 x^{2}}\right) K_{0}\left(\frac{3 \alpha}{2 x^{2}}\right)+\exp \left(-\frac{3 \alpha}{2 x^{2}}\right) K_{1}\left(\frac{3 \alpha}{2 x^{2}}\right)$.

$K_{0}(x)$ and $K_{1}(x)$ are the modified Bessel function of order 0 and 1, respectively. Even in this case, to have one and only one solution, we demand that the expression in the right hand side of Eq. (24) has a stationary point with respect to $x$ which coincides with the constant value 1. For a generic but small $\alpha$, we can expand in powers of $\alpha$ to find

$$
0=\frac{d}{d x}\left[\frac{1}{2 \pi^{2} x^{2}} f(\alpha, x)\right] \simeq \frac{20-10 \ln \left(4 x^{2} / \alpha\right)+10 \gamma_{E}+9 \ln 3}{\pi^{2} x^{2}}+O(\alpha),
$$

which has a root at

$$
\bar{x}=r_{t} E_{P}=2.973786871 \sqrt{\alpha} .
$$

Substituting $\bar{x}$ into Eq. (24), we find

$$
1=\frac{0.2423530631}{\alpha}
$$

fixing therefore $\alpha \simeq 0.242$. It is interesting to note that this value is very close to the value $\alpha=1 / 4$ used in Ref.[7] inspired by Noncommutative analysisof Ref.[6]. As in Refs. [4, 5], it is rather important to emphasize a shortcoming in the analysis carried in this section, mainly due to the technical difficulties encountered. Note that we have considered a variational approach which imposes a local analysis to the problem, namely, we have restricted our attention to the behavior of the metric function $b(r)$ at the wormhole throat, $r_{0}$. Despite the fact that the behavior is unknown far from the throat, due to the high curvature effects at or near $r_{0}$, the analysis carried out in this section should extend to the immediate neighborhood of the wormhole throat. Nevertheless it is interesting to observe that in Ref.[3] the greatest value of the wormhole throat was fixed at $r_{0} \simeq 1.16 / E_{P}$ using a regularizationrenormalization scheme. From Eq.(27), one immediately extracts $r_{0} \simeq 1.46 / E_{P}$ which is slightly larger. We have to remark that in the Noncommutative case we have only one parameter to be fixed: $\theta=7.43 \times 10^{-2} l_{P}^{2}$. On the other hand, in Gravity's Rainbow, we have more flexibility, because of the unknown functions $g_{1}\left(E / E_{P}\right)$ and $g_{2}\left(E / E_{P}\right)$. Therefore we conclude that Gravity's Rainbow offers a wider variety of examples which deserve to be explored.

\section{References}

[1] M. S. Morris and K. S. Thorne, Am. J. Phys. 56, 395 (1988)

[2] M. Visser Lorentzian Wormholes: From Einstein to Hawking (American Institute of Physics, New York, 1995)

[3] R. Garattini, Class. Quant. Grav. 22, 1105 (2005); arXiv:gr-qc/0501105

[4] R. Garattini, Class. Quant. Grav. 24, 1189 (2007), arXiv: gr-qc/0701019 R. Garattini and F. S. N. Lobo, Class. Quant. Grav. 24, 2401 (2007), arXiv:gr-qc/0701020

[5] R. Garattini and F. S. N. Lobo, Phys. Lett. B 671, 146 (2009), arXiv:0811.0919

[6] R. Garattini and P. Nicolini, Phys. Rev. D 83, 064021 (2011), arXiv:1006.5418

[7] R. Garattini and G. Mandanici, Phys. Rev. D 83, 084021 (2011), arXiv:1102.3803

[8] R. Garattini and F. S.N. Lobo, Phys. Rev. D 85024043 (2012), arXiv: 1111.5729 
